flux and gradient flow). Neglecting the change of molar volume between phases and internal stresses, the local equilibrium contribution is defined by the contribution from bulk phases (grains) and the contribution from the diffuse interface (the latter takes into account takes into account the additional energy associated with the relaxation of the diffusion flux and gradient flow).

The evolution equations for fast crystallization are written using multi-phase interpolation functions among growing poly-crystallites. The equations for diffusional mass transport and multi-phase evolution are written in a form suitable for numerical and analytical solutions $[3,4]$.

1. Galenko P., Jou D., Phys. Rev. E., 71, 046125-1-13 (2005).

2. Moelans N., Blanpain B., Wollants P., Phys. Rev. Letters, 101, 025502-1-4 (2008).

3. Nizovtseva I.G., Galenko P.K., Alexandrov D.V., Journal of Physics A: Mathematical and Theoretical, 49, 435201-1-14 (2016).

4. I.G. Nizovtseva, P.K. Galenko, Philosophical Transactions A, Royal Society, 376, 20170202-1-15 (2018).

\title{
TRAVELING WAVE SOLUTION OF PHASE FIELD MODEL FOR MULTI-GRAIN SYSTEMS
}

\author{
Galenko P. K. ${ }^{1,2}$, Nizovtseva I. G. ${ }^{1 *}$, Moelans N. ${ }^{3}$ \\ 1) Ural Federal University named after first President of Russia B.N. B.N.Yeltsin, \\ Yekaterinburg, Russia \\ ${ }^{2)}$ Friedrich-Schiller-Universität Jena, Jena, Germany \\ 3) Katholieke Universiteit Leuven, Leuven, Belgium \\ *E-mail: nizovtseva.irina@gmail.com
}

Using a new phase field model for fast polycrystalline crystallization [1], an analysis of fast and slow crystallization regimes is provided using analytical methods suggested in Refs. [2,3]. Fast regimes of crystallization are analyzed when the crystal growth velocity is of the order of or greater than the solute diffusion speed in a bulk metastable phase. The latter regime represents diffusionless (chemically partitionless) crystallization, when the grains are growing with the initial nominal concentration. In the limiting slow crystallization regime, the developing model [1] is reduced to the equations and solutions, as obtained by the quantitative and thermodynamically consistent multi-phase field model $[4,5]$.

Figure 1 shows the traveling wave velocity for the various relaxation time $\tau$ for the gradient flow of the phase field. Such a behavior of $V(\Delta G)$ is consistent with the data of numerical molecular dynamics simulation [6] and recent advancements on kinetics of fast interfaces [7]. The difference between velocities for different $\tau$ occurs at moderate and large values of driving forces, namely, at $\Delta G>0.2$. At small $\Delta G$, the influence of relaxation time $\tau$ is negligible. 
ФТИ-2019

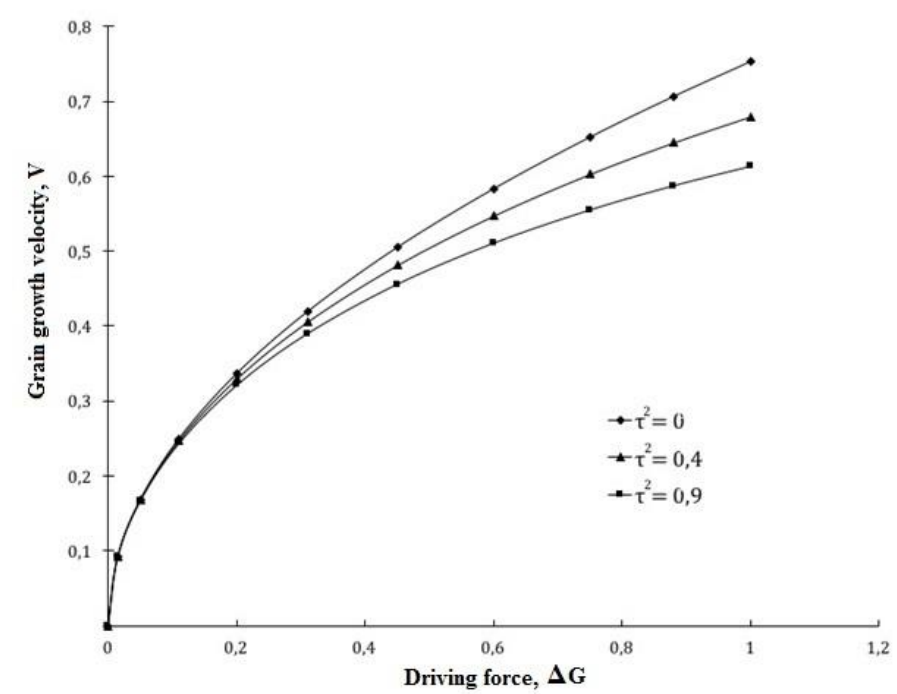

Fig. 1. Velocity of the travelling-wave solutions for the various driving forces $\Delta G$.

The obtained analytical solution in a form of traveling wave can be considered as a benchmark for the numerical solution of multi-grain problem of rapid solidification. The developed system of equation can be applied in a wide spectrum of practical issues, e.g.: rapid solidification processing in quenching from the liquid state, fast recrystallization in laser annealing, grain's refinement or coarsening in containerless technologies.

1. Galenko P., Nizovtseva I.G., Moelans N., Proceedings to the VI International Youth Scientific Conference on Physics, Technology and Innovations PTI-2019 (current) (2019)

2. Nizovtseva I.G., Galenko P.K., Alexandrov D.V., Journal of Physics A: Mathematical and Theoretical, 49, 435201-1-14 (2016).

3. Nizovtseva I.G., Galenko P.K., Philosophical Transactions A, Royal Society, 376, 20170202-1-15 (2018).

4. Moelans N., Blanpain B., P. Wollants, Phys. Rev. B, 78, 0241131-23 (2008).

5. Moelans N., Acta Mat, 59, 1077-1086 (2011).

6. Hoyt J.J., Sadigh B., Asta M., Foiles S.M., Acta Mat, 47, 3181-3187 (1999).

7. Salhoumi A., Galenko P.K., IOP Conf. Series: Mater. Sci. Eng., 192, 012014 (2017). 